\title{
Pharmacologic synergism of ocular ketorolac and systemic caffeine citrate in rat oxygen-induced retinopathy
}

\author{
Jacob V. Aranda'-3, Charles L. Cai', Taimur Ahmad', Vadim Bronshtein', Jonathan Sadeh', Gloria B. Valencia', \\ Douglas R. Lazzaro ${ }^{2,3}$ and Kay D. Beharry ${ }^{1-3}$
}

BACKGROUND: Caffeine or ketorolac decrease the risk of retinopathy of prematurity and may act synergistically to improve beneficial effect. Combination of caffeine (Caff) and ketorolac (Keto) to prevent oxygen-induced retinopathy was studied.

METHODS: Newborn rats exposed to room air (RA) or intermittent hypoxia $(\mathrm{IH})$ consisting of $12 \% \mathrm{O}_{2}$ during hyperoxia $\left(50 \% \mathrm{O}_{2}\right)$ from birth $(\mathrm{PO})$ had single daily IP injections of Caff from P0-P13 or saline; and/or ocular Keto (Acuvail, $0.45 \%$ ophthalmic solution) administered subcutaneously over the eyes from P5-P7. Pups were studied at P14 or placed in RA for recovery from IH (IHR) until P21. Eyes were examined for neovascularization, histopathology, growth factors, and VEGF-signaling genes.

RESULTS: Severe retinal damage noted during IHR in the untreated groups evidenced by hemorrhage, neovascularization, and oxygen-induced retinopathy (OIR) pathologies were prevented with Keto/Caff treatment. Keto and/or Caff treatment in $\mathrm{IH}$ also promoted retinal neural development evidenced by eye opening $(92 \%, P<0.001$ vs. $31 \%$ in the placebo-treated IH group). No corneal pathologies were noted with Keto.

CONCLUSION. Caff or Keto given individually reduced retinal neovascularization, but the two drugs given together prevented severe OIR.

$\mathbf{R}$ etinopathy of prematurity (ROP) is a neovascular disease in preterm neonates characterized by: (i) an the early vaso-obliterative phase (phase I) leading to ocular hypoxia and aberrant regulation of ocular vascular growth factors; and (ii) a later vaso-proliferative phase (phase II) of pathologic neovascularization leading to cicatricial formation, retinal detachment, and blindness $(1,2)$. Hyperoxia and intermittent hypoxia (IH) are key risk factors for ROP. Preterm newborns experience numerous IH episodes during the first few weeks of life (3-7) with fluctuations in $\mathrm{PaO}_{2}(7)$. These repeated IH episodes in recurrent apnea with exposure to hyperoxia increases the risk factor for severe ROP requiring laser treatment.
Pharmacologic interventions to prevent ROP include targeting vascular endothelial growth factor (VEGF) and its coregulators of angiogenesis. Intravitreal Bevacizumab (Avastin), an irreversible VEGF blocker is used in preterm newborns with aggressive posterior ROP. The BEAT-ROP Trial $(8,9)$ showed superiority of Avastin compared to laser. However, use of antiVEGF therapies during critical stages of development may result in unwanted long-term adverse effects $(10,11)$.

Multifactorial pathogenesis suggests that no single pharmacologic agent that may target the complex interactions of growth factors, oxidative stress, inflammatory response, and membrane disruption currently exists to prevent ROP. We propose that synergistic pharmacologic agents may correct most of the aberrant molecular and biochemical events leading to ROP. This novel approach combines Caffeine citrate (Caff) and nonsteroidal anti-inflamatory drugs (NSAIDs) to target oxidative stress and inflammatory responses, reduce the occurrence of $\mathrm{IH}$, and target some of the growth factors involved in the development of severe ROP. Caff is used worldwide for apnea prevention and to facilitate endotracheal extubation of neonates on mechanical ventilation (12). The Caffeine for Apnea of Prematurity trial involving 2,006 preterm newborns given either placebo or Caff showed decreased severe ROP and other neonatal morbidities at $18-22$ mo $(13,14)$. Studies on growth factors and morphogens such as Sonic Hedgehog (15), VEGF and hypoxia-inducible factor (HIF)-1 $\alpha$ (16), as well as matrix degrading enzymes (17), indicate that Caff exerts powerful effects on these molecular events that may lead to the observed protective effect on ROP. Prostanoids are involved in the development of oxygen-induced retinopathy (OIR) and the cross-talk between VEGF, oxidative stress, and cyclooxygenase (COX) pathways (18-23). Ketorolac (Keto) eye drops, an ophthalmic NSAID that inhibit COX, had been used safely and had decreased ROP in ketorolac treated preterm infants compared to a historical control (23).

Thus, we tested the hypothesis that the combined use of topical ocular Keto and systemic Caff exerts synergistic actions to prevent severe OIR in our rat model simulating apnea and $\mathrm{IH}$, or brief arterial oxygen desaturations in ELGANs. We 
administered Cafcit from the first day of life (P0) to P13 and/or ophthalmic ketorolac (Acuvail) from P5 to P7 during exposure to IH to determine whether drugs with complimentary actions given together act synergistically on retinal angiogenesis and OIR prevention.

\section{RESULTS}

\section{Effects on Eye Opening and Somatic Growth}

The ceacal period encompassing the time of conception to eye opening, is an indication of retinal neural circuitry maturation in rats. Rats open their eyes at P14. For this reason, we exposed the animals to $\mathrm{IH}$ from P0 to P14. This phase also coincides with phase I of ROP. Table 1 shows that at P14, eye opening occurred in 58 to $61 \%$ of pups raised in RA with placebo treatment. IH significantly decreased eye opening to 31 to $36 \%$. Keto or Caff accelerated eye opening up to 75 and $78 \%$, respectively and $92 \%$ when given together. ROP is most severe in undernourished ELGANs; thus, we examined the effects of IH on somatic growth. We determined percentage change from P0 in order to account for differences in body weight at birth. Although we pooled the litters to eliminate litter size differences, we found that larger dams delivered larger pups; so, there were variations in birth weight over the course of the experiments. Table 2 shows that weight accretion was lower in IH-exposed, placebo-treated animals during recovery from IH (IHR). Treatment with Keto and/or Caff in IH improved weight accretion ( $n=18$ pups per group).

\section{Effect on VEGF Protein}

Systemic VEGF levels (pg/ml) are presented in Table 3. VEGF levels ( $n=10$ samples per group) were significantly higher in the Keto/Caff groups exposed to IH. Retinal and choroidal

Table 1. Eye opening at P14

\begin{tabular}{lccc}
\hline & \multicolumn{3}{c}{ P14 } \\
\cline { 2 - 4 } Sal/Sal: & Left eye & Right eye & Both eyes \\
RA & $21(58 \%)$ & $22(61 \%)$ & $21(61 \%)$ \\
IH & $13(36 \%)$ & $11(31 \%)^{5}$ & $11(31 \%)^{\S}$ \\
Keto/Sal: & & & \\
RA & $21(58 \%)$ & $24(67 \%)$ & $21(58 \%)$ \\
IH & $33(92 \%)^{\ddagger, \S}$ & $27(75 \%)^{\ddagger}$ & $27(75 \%)^{\ddagger \S}$ \\
Sal/Caff: & & & \\
RA & $27(75 \%)^{* *}$ & $27(75 \%)$ & $27(75 \%)$ \\
IH & $28(78 \%)^{\ddagger}$ & $25(78 \%)^{\ddagger}$ & $25(78 \%)^{\ddagger}$ \\
IP Caffeine + Ocular Ketorolac & & & \\
RA & $34(94 \%)^{* *}$ & $30(83 \%)^{* *}$ & $30(83 \%)^{* *}$ \\
IH & $33(92 \%)^{\ddagger}$ & $33(92 \%)^{\ddagger}$ & $33(92 \%)^{\ddagger}$ \\
\hline
\end{tabular}

At P14, all eyes were assessed for eye opening. Animals in the same oxygen condition and drug treatment group were pooled for a total of $n=36$. ${ }^{*} P<0.01 \mathrm{vs}$. IP Sal/Sal RA; ${ }^{\ddagger} P<0.01 \mathrm{vs}$. IP Sal $/ \mathrm{Sal} I \mathrm{H} ;{ }^{\S} P<0.01 \mathrm{vs}$. RA.

$\mathrm{IP}$, intraperitoneal; $\mathrm{IH}$, intermittent hypoxia; $I \mathrm{HR}$, recovery from $\mathrm{IH}$; RA, room air. Ocular saline+IP saline (Sal/Sal); Ocular ketorolac+IP saline (Keto/Sal); Ocular saline+IP caffeine (Sal/Caff); Ocular ketorolac+IP caffeine (Keto/Caff).
VEGF levels (pg/mg protein) are presented in Figures 1 and 2 (panels a and d), respectively ( $n=6$ samples per group). At P14 (Figure 1a), Sal/Caff treatment in IH decreased retinal VEGF, as did treatment with Keto/Caff in IH albeit to a lesser degree. At P21, Keto/Sal and Sal/Caff decreased retinal VEGF during IHR (Figure 1d). In the choroid, VEGF was decreased in the placebo IH group, and elevated with Keto/Sal and Keto/ Caff in IH (Figure 2a). The response was sustained during IHR (Figure 2d).

\section{Effect on sVEGFR-1}

Systemic sVEGFR-1 levels (pg/ml), presented in Table 3, show decreased VEGFR-1 with Keto/Sal and Keto/Caff in $\mathrm{RA}$ at P14; and with all treatment groups during $\mathrm{IH}(n=10$ samples per group). Retinal and choroidal sVEGFR-1 levels (pg/mg protein) are presented in Figures 1 and 2 (panels b and e), respectively ( $n=6$ samples per group). At P14 (IH), retinal sVEGFR-1 levels were significantly decreased in the Sal/Sal controls and elevated in the treated groups (Figure 1b). At P21 (IHR), the levels further declined in the $\mathrm{Sal} / \mathrm{Sal}$ controls and remained elevated in the treated groups (Figure 1e). Choroidal responses in IH and IHR were similar to those noted in the retina (Figure $2 \mathrm{~b}, \mathrm{e}$ ).

\section{Effect on IGF-I}

Systemic IGF-I levels (pg/mL), presented in Table 3, show elevated IGF-I levels in response to Keto/Sal and/or Keto/ Caff in IH ( $n=10$ samples per group). These effects remained sustained at P21. Retinal and choroidal IGF-I levels (pg/mg protein) are presented in Figures 1 and 2 (panels $c$ and $\mathrm{f}$ ), respectively ( $n=6$ samples per group). In the retina, IGF-I levels declined in the Sal/Sal controls during IH (P14) but were elevated in all treated groups (Figure 1c). At P21 (IHR), only those animals treated with Keto/Caff in RA and IH had higher retinal IGF-I levels (Figure 1f). Choroidal IGF responded similarly to that of the retina during IH (Figure 2c). At P21, choroidal IGF-I levels were elevated in all treated groups during IHR (Figure 2f).

\section{Effect on HIF1 $\alpha$}

Retinal and choroidal HIF $_{1 \alpha}\left(\mathrm{pg} / \mathrm{mg}\right.$ protein) and 8 -isoPGF ${ }_{2 \alpha}$ (pg/mg protein) levels are presented in Figure 3 ( $n=6$ samples per group). Retinal $\mathrm{HIF}_{1 \alpha}$ levels during IH are presented in Figure $3 \mathrm{a}$ and during IHR in Figure $3 \mathrm{e}$. Choroidal HIF $\mathrm{H}_{1 \alpha}$ levels during $\mathrm{IH}$ are presented in Figure $3 \mathrm{c}$ and during IHR in Figure 3g. At P14, retinal $\mathrm{HIF}_{1 \alpha}$ increased in the Sal/Sal group exposed to IH. Keto/Sal also increased retinal $\mathrm{HIF}_{1 \alpha}$ in $\mathrm{RA}$ while Keto/Caff treatment decreased $\mathrm{HIF}_{1 \alpha}$ in IH (Figure 3a). During IHR, a more profound elevation in retinal $\mathrm{HIF}_{1 \alpha}$ was noted in the Sal/Sal group, suggesting IH-induced sustained $\mathrm{HIF}_{1 \alpha}$ stabilization. Treatment with Keto/Sal and Sal/Caff in $\mathrm{IH}$ decreased retinal $\mathrm{HIF}_{1 \alpha}$ (Figure $3 \mathrm{e}$ ). In the choroid, treatment with Caff and/or Keto did not appreciably change $\mathrm{HIF}_{1 \alpha}$ levels during $\mathrm{IH}$ (Figure $3 \mathrm{c}$ ), or IHR (Figure 3g). 


\section{Articles | Aranda e tal}

Table 2. Weight accretion and percentage change in body weight and linear growth from birth

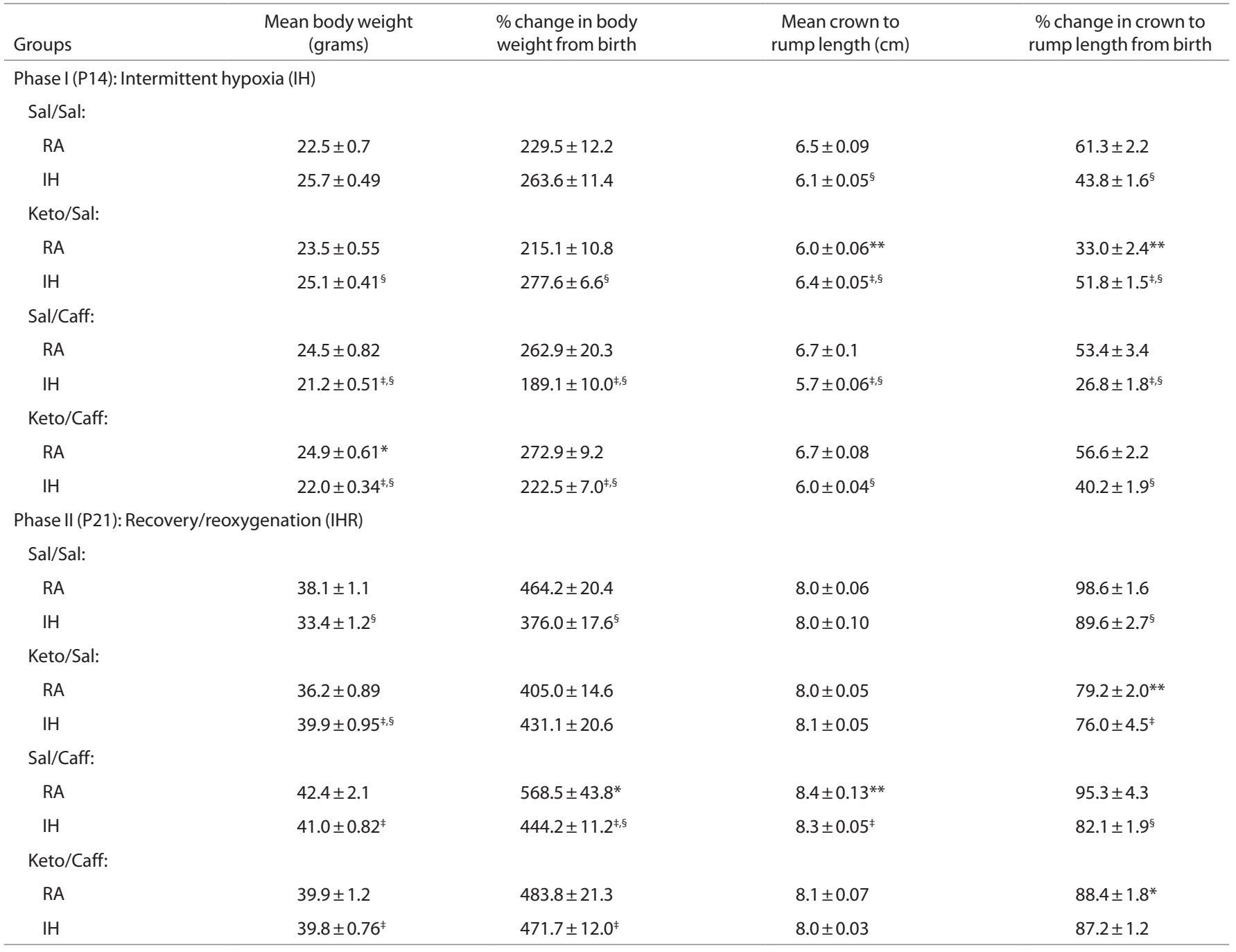

Data are mean $\pm S D, n=18$ pups per group $\left({ }^{*} P<0.05,{ }^{* *} P<0.01 \mathrm{vs}\right.$. Sal/Sal RA; ${ }^{\ddagger} P<0.01 \mathrm{vs}$. Sal/Sal IH; ${ }^{\S} P<0.01 \mathrm{vs}$. RA; $n=18 /$ group).

RA, room air

Ocular saline+IP saline (Sal/Sal); Ocular ketorolac+IP saline (Keto/Sal); Ocular saline+IP caffeine (Sal/Caff); Ocular ketorolac+IP caffeine (Keto/Caff).

\section{Effect on 8-isoPGF2 $\alpha$}

8 -isoPGF ${ }_{2 \alpha}$ levels (biomarker for oxidative stress) in retina and choroid, are presented in Figure $3(n=6$ samples per group). Retinal 8 -isoPGF ${ }_{2 \alpha}$ levels during $\mathrm{IH}$ are presented in Figure $3 \mathbf{b}$ and during IHR in Figure 3 f. Choroidal 8-isoPGF ${ }_{2 \alpha}$ levels during $\mathrm{IH}$ are presented in Figure $3 \mathrm{~d}$ and during IHR in Figure 3h. At P14, retinal 8-isoPGF ${ }_{2 \alpha}$ levels increased in the $\mathrm{Sal} / \mathrm{Sal}$ group exposed to $\mathrm{IH}$ (Figure $3 \mathbf{b}$ ) and remained elevated during IHR at P21 (Figure 3f). Treatment with Keto and/ or Caff significantly reversed this response. A similar response pattern was noted in the choroid during $\mathrm{IH}$ (Figure 3d) and IHR (Figure 3h), although there was an increase with Sal/Caff treatment in RA.

\section{Effect on Retinal Neovascularization}

A representative ADPase-stained retinal flatmounts from RA controls (panels a and b), Sal/Sal IHR (panels $c$ and d) and Keto/Caff IHR (panels e and f) groups at P21 are presented in Figure 4. Flatmounts from animals at P14 (IH) and individual treatment with Keto/Sal or Sal/Caff are presented in Supplementary Figure S1 online. The figures show normal retinal vascularization at the optic disk (panel a) and periphery (panel b) in the RA controls. In contrast, punctate hemorrhages (arrow) were noted around the optic disk (panel c), as well as severe retinal hemorrhage, dilated vessels, aberrant angiogenesis, and vascular tufts (arrows) in the peripheral retinas (panel d) in the placebo-treated (Sal/Sal) groups. Treatment with combined Keto/Caff showed reduced vascular density and size, and a wide avascular zone at the periphery with no evidence of hemorrhage, tortuosity or vascular tufts at the optic disk (panel e) and peripheral (panel f) retina. Images are $4 \times$ magnification.

\section{Histopathology and Immunofluorescence}

Representative $\mathrm{H} \& \mathrm{E}$-stained retinas and corneas from RA controls (panels a and d), Sal/Sal IHR (panels b1, b2, and e) and 
Table 3. Serum levels of VEGF, sVEGFR-1, and IGF-I

\begin{tabular}{|c|c|c|c|c|}
\hline Groups & $\mathrm{Sal} / \mathrm{Sal}$ & Keto/Sal & Sal/Caff & Keto/Caff \\
\hline \multicolumn{5}{|l|}{ P14 (phase I): RA } \\
\hline VEGF (pg/ml) & $15.0 \pm 1.12$ & $13.8 \pm 1.8$ & $19.8 \pm 2.9$ & $28.7 \pm 1.9^{* *}$ \\
\hline IGF-I (pg/ml) & $4,439.0 \pm 158.2$ & $7,881.1 \pm 261.2^{* *}$ & $4,465.8 \pm 225.4$ & $9,205.1 \pm 198.9^{* *}$ \\
\hline \multicolumn{5}{|l|}{ P14 (phase I): IH } \\
\hline IGF-I (ng/ml) & $7,736.7 \pm 133.8^{\S}$ & $9,286.4 \pm 169.9^{\ddagger, \S}$ & $8,267.9 \pm 268.7^{\S}$ & $7,953.1 \pm 497.0$ \\
\hline \multicolumn{5}{|l|}{ P21 (phase II): RA } \\
\hline VEGF (pg/ml) & $0.51 \pm 0.1$ & $28.2 \pm 3.2^{* *}$ & $4.4 \pm 1.5$ & $26.7 \pm 3.4^{* *}$ \\
\hline sVEGFR-1 (pg/ml) & $88.4 \pm 25.7$ & $231.2 \pm 28.8^{* *}$ & $209.0 \pm 35.6^{*}$ & $245.6 \pm 25.5^{* *}$ \\
\hline sVEGFR-1 (pg/ml) & $128.4 \pm 29.3$ & $250.2 \pm 26.6^{\ddagger}$ & $179.0 \pm 24.1^{\ddagger}$ & $315.8 \pm 35.6^{\ddagger}$ \\
\hline IGF-I (ng/ml) & $7,712.6 \pm 145.5^{5}$ & $8,840.0 \pm 102.7^{\ddagger}$ & $9,298.3 \pm 165.8^{\ddagger, \S}$ & $9,463.0 \pm 161.1^{\ddagger}$ \\
\hline
\end{tabular}

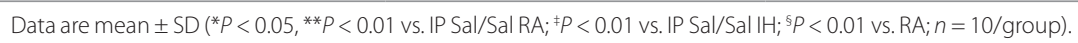

IP, intraperitoneal; RA, room air. Ocular saline+IP saline (Sal/Sal); Ocular ketorolac+IP saline (Keto/Sal); Ocular saline+IP caffeine (Sal/Caff); Ocular ketorolac+IP caffeine (Keto/Caff).
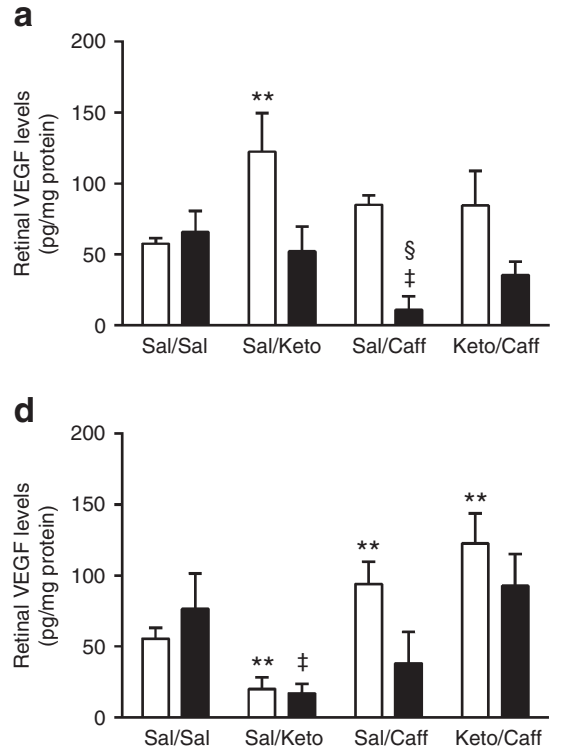
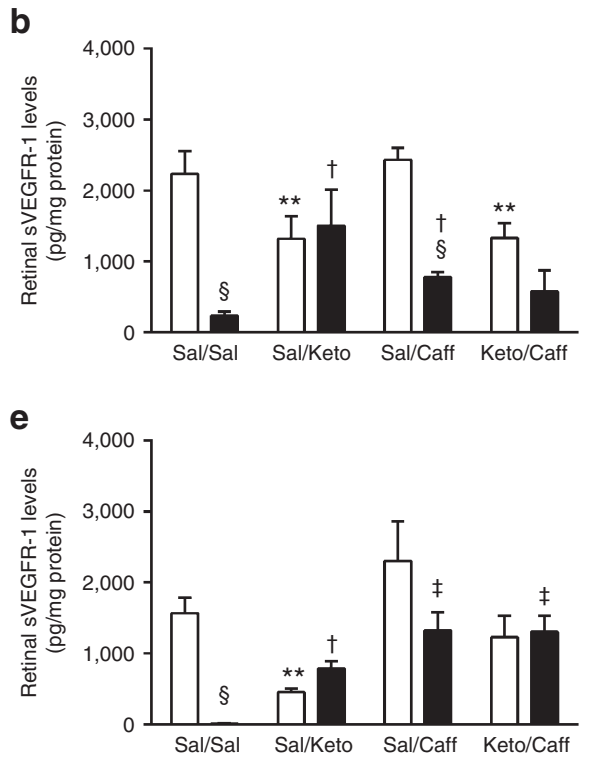

C

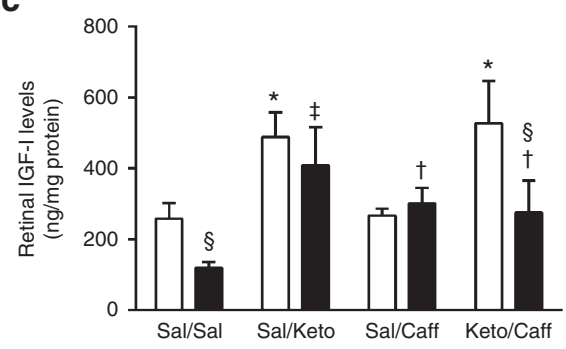

f

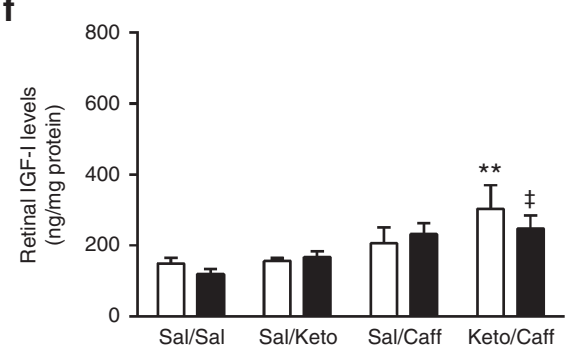

Figure 1. Effects of Keto and/or Caff on retinal VEGF, sVEGFR-1, and IGF-I. The upper panel (a-c) are data at P14 (IH), and the lower panel (d-f) are data at P21 (IHR). The white bars represent the room air (RA) controls and the black bars represent the intermittent hypoxia (IH) at P14 or recovery from IH (IHR) at P21. Data are presented as mean \pm SEM ( $n=6$ samples/group). ${ }^{*} P<0.01 ;{ }^{* *} P<0.01 \mathrm{vs.} \mathrm{Sal/Sal} \mathrm{RA;}{ }^{+} P<0.05 ;{ }^{\ddagger} P<0.01 \mathrm{vs}$. Sal $/ \mathrm{Sal} \mathrm{IH} ;{ }^{\text {s }} P<0.01 \mathrm{vs}$. RA.

Keto/Caff IHR (panels $\mathrm{c}$ and $\mathrm{f}$ ) groups at P21 are presented in Figure 5. Corresponding images of the retinal layers and corneas at P21 (IHR) for Keto/Sal or Sal/Caff treatment are presented in Supplementary Figure S2 online. Immunofluorescence staining for corneal COX-2 (green) is presented alongside the corresponding corneal $\mathrm{H} \& \mathrm{E}$ stains. The retinal layers (panel a) and corneas (panel d) of the RA controls appeared normal. The placebo-treated IHR groups severe nerve fiber layer (NFL) vacuolization and/or loss, GCL (ganglion cell layer) nuclear atrophy, and $\mathrm{P}$ (photoreceptor) degeneration (panel b1, arrows, $40 \times$ magnification), as well as increased neovascularization penetrating the vitreous cavity (panel b2, arrow, $60 \times$ magnification). In addition, there was evidence of corneal degeneration (panel e, arrow) and increased COX-2 immunostaining (panel e, arrow). In the Keto/Caff group exposed to IH, daily inspection of the eyes from the time of administration of Keto to the time of euthanasia showed no adverse effects. This was confirmed by histopathological examination during IHR which showed no 

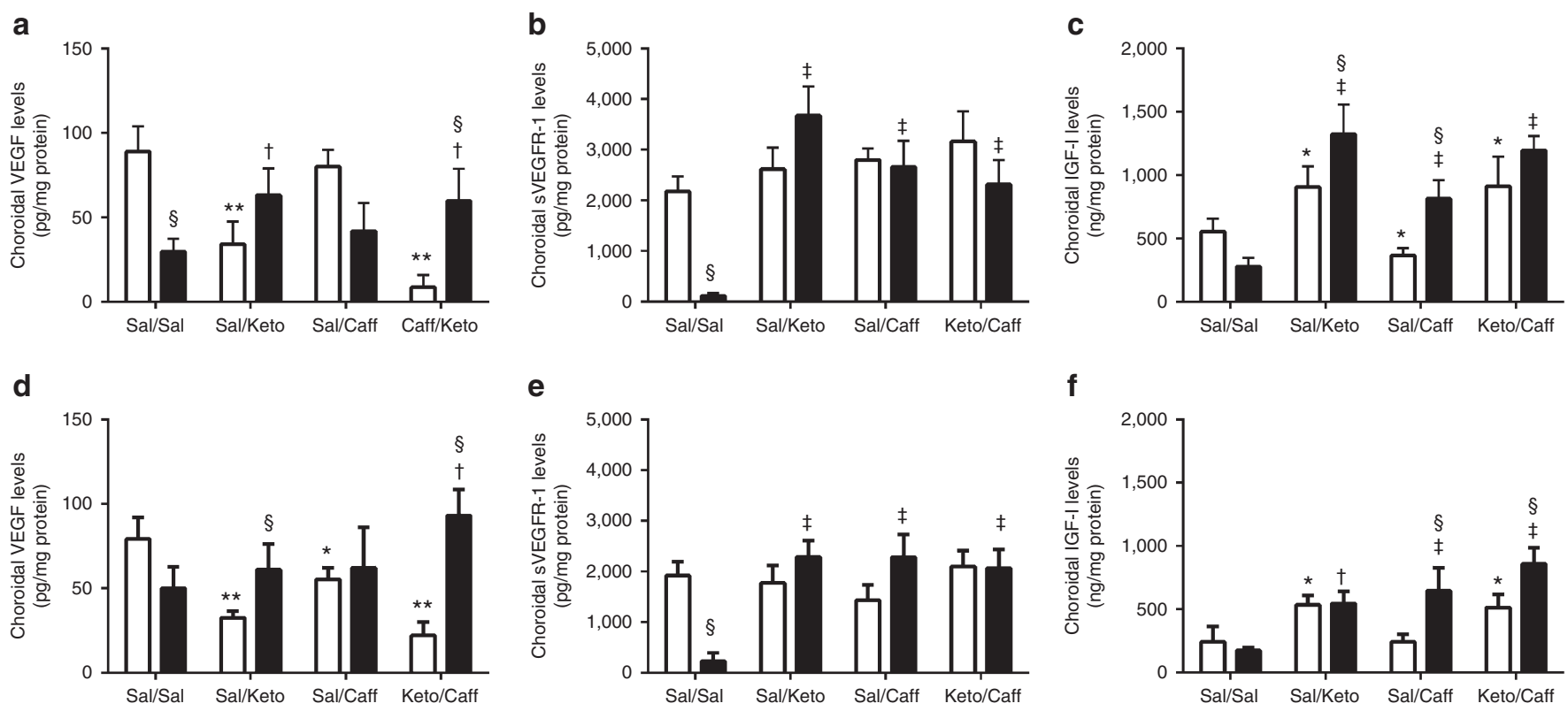

Figure 2. Effects of Keto and/or Caff on choroidal VEGF, sVEGFR-1, and IGF-I. The upper panel (a-c) are data at P14 (IH), and the lower panel (d-f) are data at P21 (IHR). The white bars represent the room air (RA) controls and the black bars represent the intermittent hypoxia (IH) at P14 or recovery from IH (IHR) at P21. Data are presented as mean \pm SEM ( $n=6$ samples/group). ${ }^{*} P<0.01 ;{ }^{* *} P<0.01$ vs. Sal/Sal RA; ${ }^{\dagger} P<0.05 ;{ }^{\ddagger} P<0.01 \mathrm{vs}$. Sal/Sal IH; ${ }^{\S} P<0.01 \mathrm{vs}$. RA.
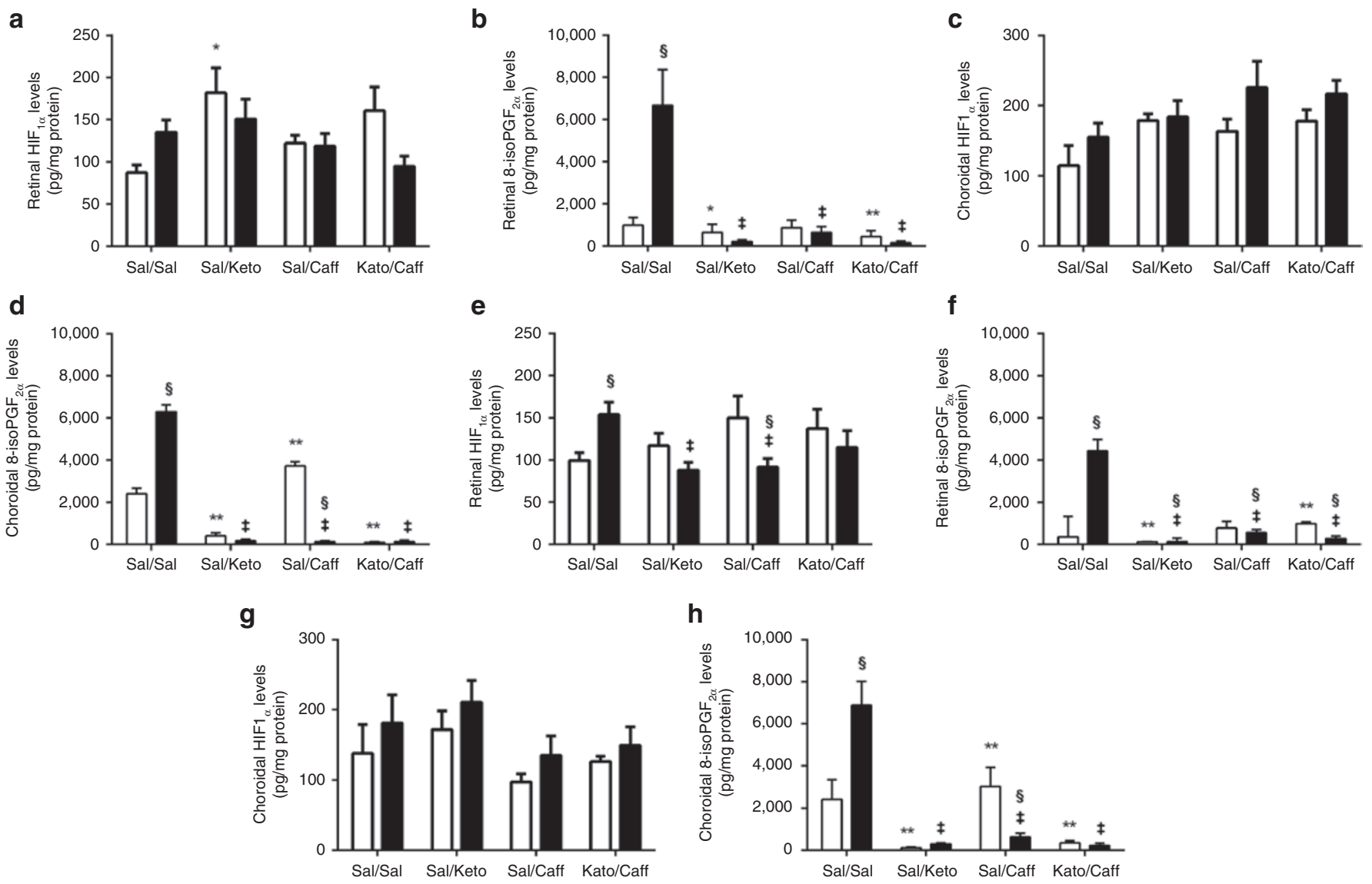

Figure 3. Effects of Keto and/or Caff on retinal and choroidal $\mathrm{HIF}_{1 \alpha}$ and 8-isoPGF ${ }_{2 \alpha}$. The upper panel (a-d) are data at P14 (IH), and the lower panel (e-h) are data at P21 (IHR). The white bars represent the room air (RA) controls and the black bars represent the intermittent hypoxia (IH) at P14 or recovery from IH (IHR) at P21. Retinal HIF $_{1 \alpha}$ are panels $\mathbf{a}$ and $\mathbf{e}$; choroidal HIF $_{1 \alpha}$ are panels $\mathbf{c}$ and $\mathbf{g}$; retinal 8-isoPGF $\mathrm{F}_{2 \alpha}$ are panels $\mathbf{b}$ and $\mathbf{f}$; and choroidal 8-isoPGF ${ }_{2 \alpha}$ are panels $\mathbf{d}$ and $\mathbf{h}$ at P14 (upper panel) and P21 (lower panel). Groups are as described in Figure 1. Data are presented as mean \pm SEM ( $n=6$ samples/group). ${ }^{*} P<0.01 ;{ }^{* *} P<0.01$ vs. Sal $/ \mathrm{Sal} \mathrm{RA} ;{ }^{*} P<0.01 \mathrm{vs}$. Sal $/ \mathrm{Sal} \mathrm{IH} ;{ }^{5} P<0.01$ vs. RA. 


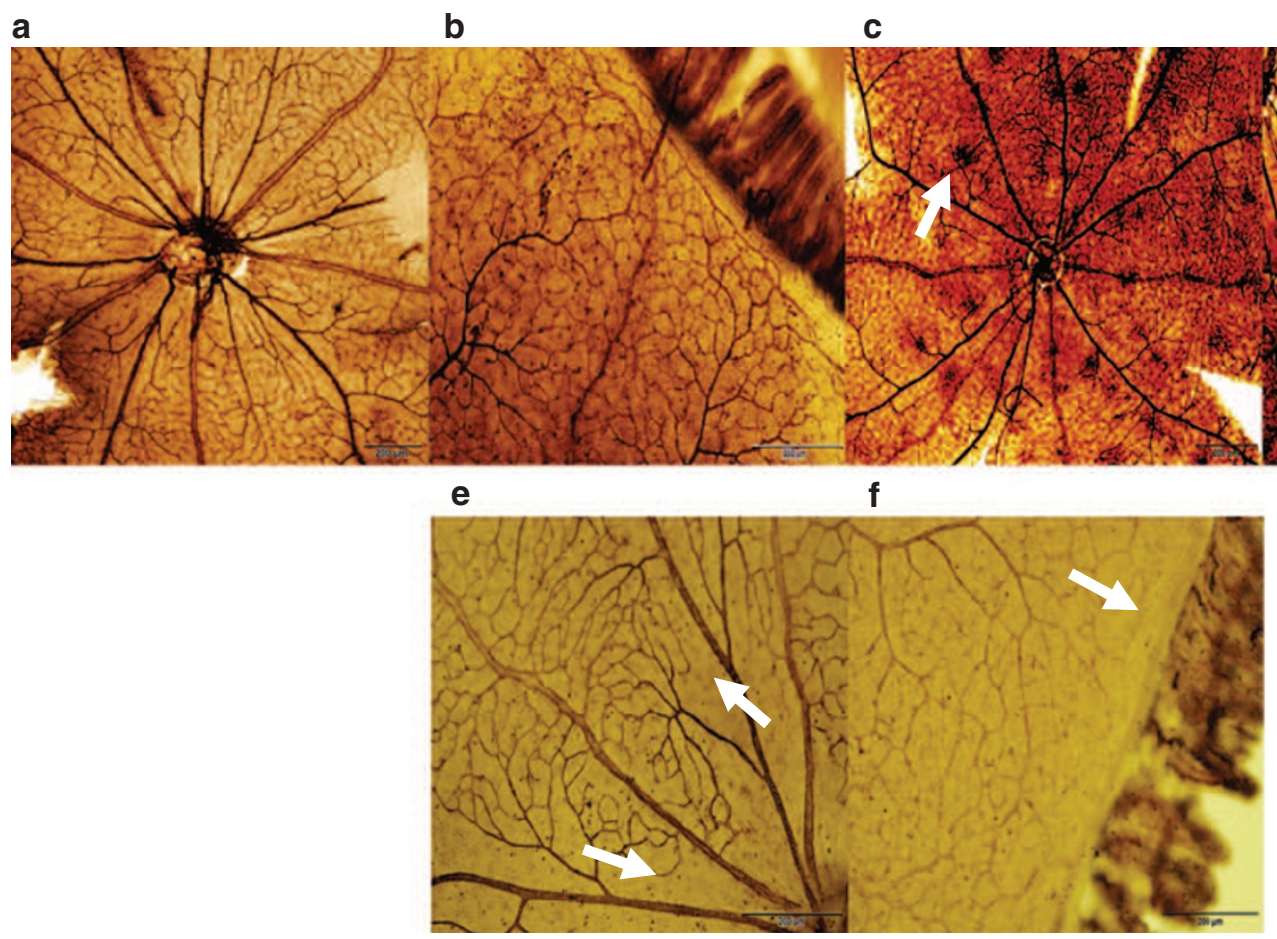

Figure 4. Retinal flatmounts of P21 RA controls, Sal/Sal IHR, and Keto/Caff-treated IHR retinas. Panels a and b represent RA controls, panels c and $\mathbf{d}$ represent Sal/Sal IHR groups, and panels $\mathbf{e}$ and $\mathbf{f}$ represent Caff/Keto-treated IHR groups. RA images show normal retinal vasculature in the optic disk (panel a) and periphery (panel b). The placebo-treated IHR groups show punctate hemorrhages at the optic disk (panel c, arrow), and neonvascularization, vascular tufts, and tortuous, dilated vessels consistent with severe OIR at the periphery (panel d, arrows). Caff/Keto reduced the severity of OIR with evidence of capillary dropout at the optic disk (panel e, arrow) and periphery (panel f, arrows). Images are $4 \times$ magnification, bar scale is $200 \mu \mathrm{m}$.
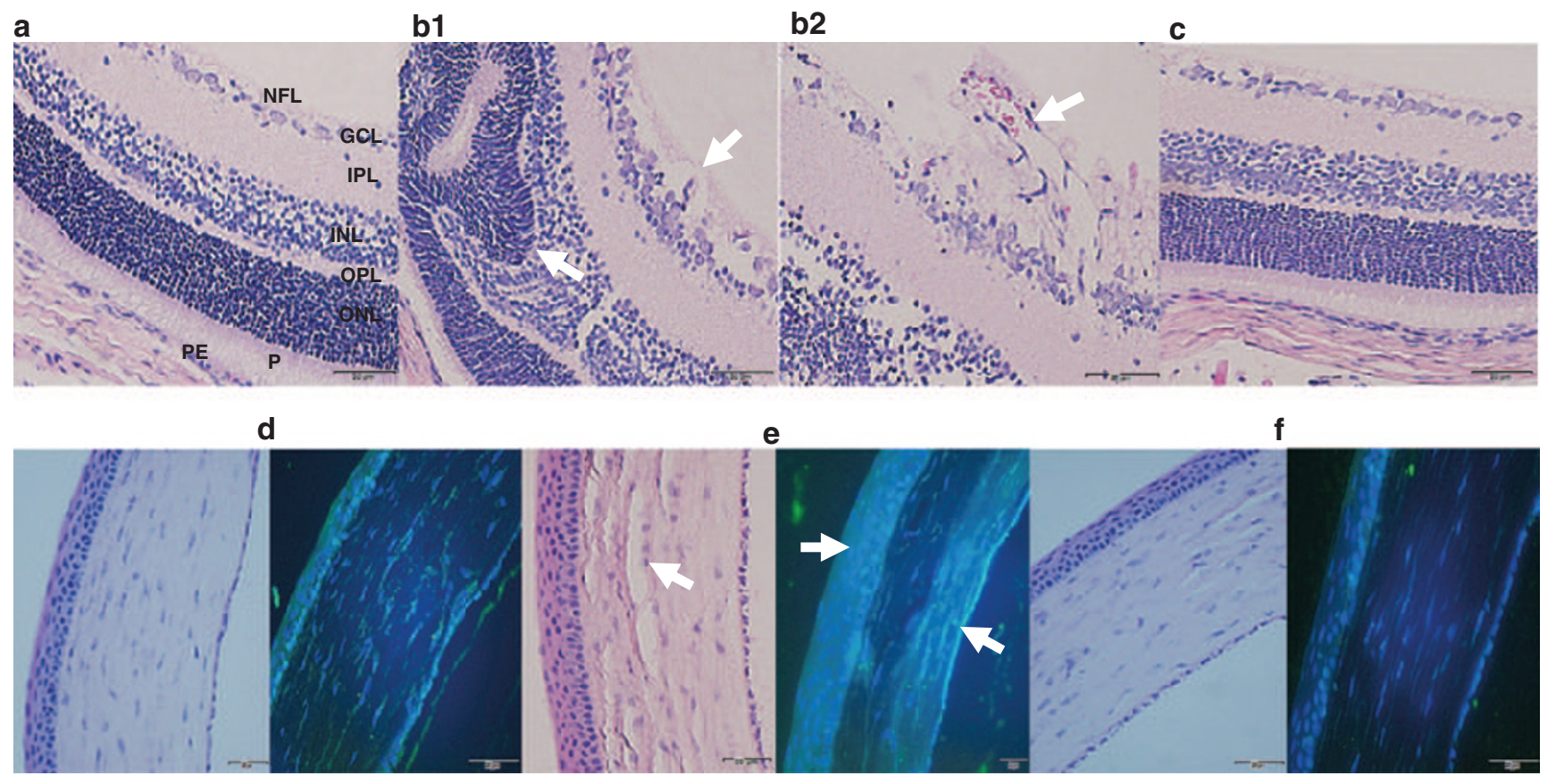

Figure 5. H\&E and COX-2 immunofluorescence of retinal layers from P21 RA controls, Sal/Sal IHR, and Keto/Caff-treated IHR groups. Panels a and d represent RA controls, panels b1, b2 and e represent Sal/Sal IHR groups, and panels $\mathbf{c}$ and $\mathbf{f}$ represent Caff/Keto-treated IHR groups. RA control retina and cornea appear normal. Sal/Sal IHR retinas show neovascularization retinal cells invading the vitreous fluid (panel b1, 40× and panel b2, 60X, arrows), retinal folding, and photoreceptor abnormalities (panel b1, arrows), as well as corneal degeneration with increased COX-2 immunostaining (e, arrows). Treatment with Caff/Keto reduced the effects (panels $\mathbf{c}$ and f). Bar scale is $20 \mu \mathrm{m}$. NFL (nerve fiber layer); GCL (ganglion cell layer); IPL (inner plexiform layer); INL (inner nuclear layer); OPL (outer plexiform layer), ONL (outer nuclear layer); P (photoreceptors); PE (pigmented epithelium); C (choroid). 


\section{Articles | Arandaetal.}

disruptions of cornea, toxic keratolysis nor retinal abnormalities (panels $\mathrm{c}$ and f). Treatment with Keto/Caff also decreased COX-2 corneal immunostaining (panel f) compared to RA (panel d) and IHR (panel e) groups.

Figure 6 represents immunofluorescence staining of the retinal layers for $\mathrm{HIF}_{1 \alpha}$ (red) VEGF (red), VEGFR-2 (red) and COX-2 (green) counterstained with DAPI (blue) from RA controls (panel a), Sal/Sal IHR (panel b), and Keto/Caff IHR (panel c) groups at P21. Images at P14 (IH) and individual treatment with either Keto/Sal or Sal/Caff are presented in Supplementary Figure S3 online. Retinas from RA controls (panel A) show some HIF staining in the GCL, INL, and P layers; VEGF staining in the IPL, OPL, and P layers; VEGFR-2 staining in the IPL, OPL, ONL, and P layers; and COX-2 staining in the NFL, IPL, $\mathrm{OPL}$, and $\mathrm{P}$ layers. In the placebo-treated IHR groups there was increased $\mathrm{HIF}_{1 \alpha}, \mathrm{VEGF}$, and COX-2 immunostaining (panel B). $\mathrm{HIF}_{1 \alpha}$ was predominantly stained in the cells invading the vitreous fluid, the retinal pigmented epithelium (RPE), and choroid (arrows). VEGF was increased in the NFL, GCL, IPL, OPL, ONL, and $\mathrm{P}$, and COX- 2 in the NFL, GCL, OPL, and P. Treatment with Keto/Caff moderately reduced VEGF and COX-2, but not $\mathrm{HIF}_{1 \alpha}$ in the $\mathrm{P}$ (panel c). Images are $40 \times$ magnification.

\section{Effect on Retinal VEGF Signaling}

Genes involved in VEGF signaling were selected from a panel of 84 genes and presented in Tables 4 (P14, IH) and Table 5 (P21, IHR). Data are fold expression compared to RA placebo controls (Sal/Sal) and were examined in retinas from three different animals in each group. Genes that were greater than fivefold up- or downregulated are presented in red font. At P14, significant changes in gene expression were noted only in the Sal/Sal IH and Keto/Caff IH groups. During the IHR phase, treatment with Keto and/or Caff resulted in major changes in retinal gene expression, particularly genes involved in proliferation, differentiation, and inflammation. Caff appeared to have significant effects on VEGFR-3.

\section{DISCUSSION}

This study demonstrated that Keto or Caff given alone reduced retinal neovascularization, but given together may prevent severe OIR in this neonatal rat model. Co-administration of Keto with Caff also enhanced maturation of the retinal neural circuitry more effectively than when administered individually. Moreover, Keto and Caff exerted synergistic or additive effects on retinal and serum growth factors including IGF-I and VEGF. These neonatal rat data may be useful in the development of pharmacotherapies targeted at prevention of ROP in the preterm newborn. Although the overall incidence of ROP has decreased, it still remains high with threshold ROP occurring in 5\% of ELGANs in the United States, with as much as $30 \%$ of them becoming blind (24). Our neonatal rat OIR model closely simulates frequent $\mathrm{IH}$ episodes experienced by ELGANs. Using this model, we have previously shown that $\mathrm{IH}$ was associated with a more severe form of OIR (25-29) similar to ELGANs $(4,5)$. We report here that ocular Keto and systemic Caff together exert a synergistic protective effect on OIR. Previous studies showed

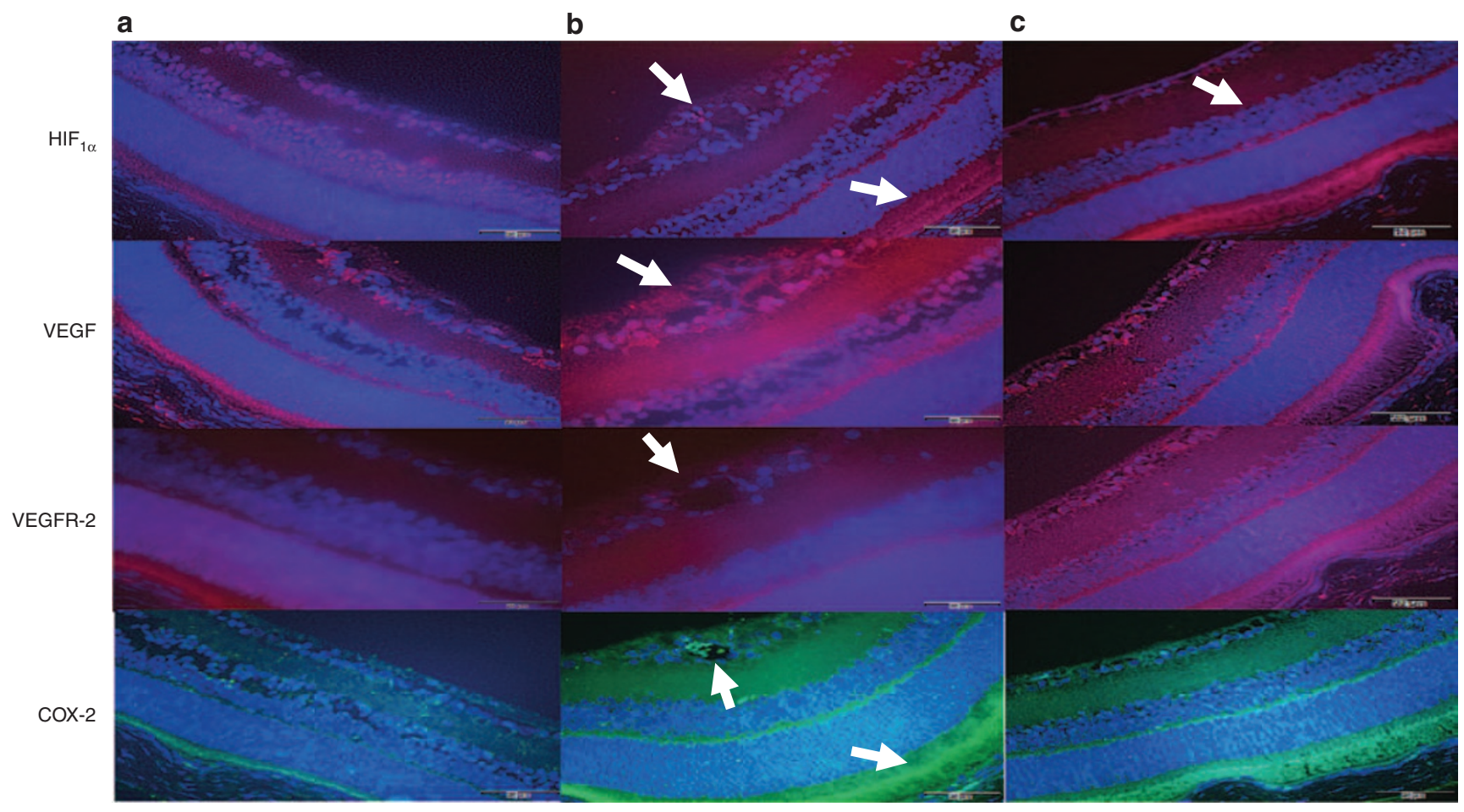

Figure 6. Immunofluorescence of $\mathrm{HIF}_{1 \alpha^{\prime}}$ VEGF, VEGFR-2, and COX-2 in retinal layers from P21 RA controls, Sal/Sal IHR, and Keto/Caff IHR groups. Panel a represents RA controls, panel $\mathbf{b}$ represents Sal/Sal IHR groups, and panel $\mathbf{c}$ represents Caff/Keto IHR groups. Layers are described in Figure 5a. Compared to RA controls, Sal/Sal IHR retinas abundant VEGF and COX-2 immunostaining in the retinal cells invading the vitreous fluid (arrow), and increased HIF1 $\alpha$ and COX-2 immunostaining in the P and C (arrow). Keto/Caff treatment decreased VEGF and COX-2, but not HIF1 $\alpha$ in the P and C (arrow). Images are 40X magnification, bar scale is $20 \mu \mathrm{m}$. 
Table 4. Fold change in retinal VEGF signaling genes at P14

\begin{tabular}{|c|c|c|c|c|c|c|c|}
\hline Genes of interest & Sal/Sal IH & Keto/Sal RA & Keto/Sal IH & Sal/Caff RA & Sal/CaffIH & Keto/Caff RA & Keto/Caff IH \\
\hline COX-2 & $13.5 \pm 0.04$ & $-1.4 \pm 0.37$ & $-1.2 \pm 0.61$ & $1.4 \pm 0.43$ & $1.6 \pm 0.26$ & $1.3 \pm 0.55$ & $2.0 \pm 0.14$ \\
\hline eNOS & $13.0 \pm 0.07$ & $1.2 \pm 0.6$ & $-1.1 \pm 0.7$ & $1.1 \pm 0.7$ & $-1.6 \pm 0.4$ & $-1.2 \pm 0.82$ & $9.7 \pm 0.14$ \\
\hline Mapk11 & $-20.3 \pm 0.01$ & $-1.0 \pm 0.83$ & $-1.5 \pm 0.09$ & $1.1 \pm 0.47$ & $-1.6 \pm 0.36$ & $-1.3 \pm 0.4$ & $-1.2 \pm 0.25$ \\
\hline Nfat5 & $14.5 \pm 0.02$ & $-1.7 \pm 0.08$ & $-1.6 \pm 0.84$ & $-1.1 \pm 0.87$ & $-1.9 \pm 0.01$ & $-1.5 \pm 0.07$ & $-4.5 \pm 0.14$ \\
\hline Pdgfc & $1.5 \pm 0.33$ & $1.7 \pm 0.15$ & $1.2 \pm 0.5$ & $1.8 \pm 0.24$ & $1.8 \pm 0.16$ & $1.3 \pm 0.6$ & $9.8 \pm 0.03$ \\
\hline Pgf & $-2.5 \pm 0.01$ & $1.4 \pm 0.15$ & $1.0 \pm 0.69$ & $1.6 \pm 0.08$ & $1.4 \pm 0.29$ & $-1.1 \pm 0.99$ & $7.4 \pm 0.13$ \\
\hline Pik3r5 & $-47.3 \pm 0.002$ & $1.4 \pm 0.14$ & $-1.0 \pm 0.90$ & $1.6 \pm 0.04$ & $1.4 \pm 0.14$ & $1.4 \pm 0.22$ & $5.4 \pm 0.13$ \\
\hline Pla2g12a & $-29.8 \pm 0.11$ & $1.6 \pm 0.04$ & $1.3 \pm 0.99$ & $1.7 \pm 0.22$ & $1.4 \pm 0.64$ & $1.1 \pm 0.70$ & $3.3 \pm 0.14$ \\
\hline Plcg2 & $-13.0 \pm 0.004$ & $1.4 \pm 0.05$ & $1.1 \pm 0.42$ & $1.3 \pm 0.14$ & $1.4 \pm 0.08$ & $-1.1 \pm 0.95$ & $2.9 \pm 0.12$ \\
\hline VEGF-B & $6.2 \pm 0.03$ & $1.4 \pm 0.49$ & $1.0 \pm 0.87$ & $1.7 \pm 0.28$ & $1.8 \pm 0.20$ & $1.8 \pm 0.23$ & $14.2 \pm 0.14$ \\
\hline VEGF-C & $-3.6 \pm 0.11$ & $-1.8 \pm 0.11$ & $-1.8 \pm 0.32$ & $-1.2 \pm 0.34$ & $-1.8 \pm 0.11$ & $-1.4 \pm 0.20$ & $4.0 \pm 0.15$ \\
\hline VEGFR-1 & $12.3 \pm 0.07$ & $1.4 \pm 0.17$ & $-1.0 \pm 0.99$ & $2.1 \pm 0.01$ & $-1.4 \pm 0.45$ & $-1.2 \pm 0.99$ & $2.8 \pm 0.13$ \\
\hline VEGFR-2 & $-1.4 \pm 0.37$ & $-1.6 \pm 0.24$ & $-2.5 \pm 0.09$ & $-1.0 \pm 0.71$ & $-1.8 \pm 0.18$ & $-2.0 \pm 0.14$ & $-2.3 \pm 0.09$ \\
\hline VEGFR-3 & $-1.4 \pm 0.43$ & $1.8 \pm 0.45$ & $1.3 \pm 0.87$ & $2.0 \pm 0.35$ & $-1.0 \pm 0.75$ & $1.1 \pm 0.98$ & $19.0 \pm 0.13$ \\
\hline
\end{tabular}

Genes of interest (in alphabetical order): COX, cyclooxygenase-2; eNOS, endothelial nitric oxide synthase; Hsp, heat shock protein; Mapk11, mitogen-activated protein kinase 11; Nfat5, nuclear factor of activated T-cells 5; NP, neuropilin-1; Pdgfc, platelet-derived growth factor c; pgf, placental growth factor; Pik3r5, phosphoinositide-3-kinase regulatory subunit 5; Pla2g $12 a$, phospholipase A2, group XIIA; PIcg2, phospholipase C, gamma 2; Ppp3r2, protein phosphatase 3 regulatory subunit B, beta isoform; Prkca, protein kinase C al pha; VEGF, vascular endothelial growth factor)-A, -B, C; VEGFR (vascular endothelial growth factor receptor)-1, -2,-3. Data are mean \pm SD (n=3/group). Data are compared to Sal/Sal in RA. Room Air (RA); Intermittent Hypoxia $(\mathrm{IH})$; IP (intraperitoneal). Minus value = downregulated. Ocular saline+IP saline (Sal/Sal); Ocular ketorolac+IP saline (Keto/Sal); Ocular saline+IP caffeine (Sal/Caff); Ocular ketorolac+IP caffeine (Keto/Caff).

that Caff decreases severe ROP $(13,14)$ and NSAIDs may prevent ROP in humans (21-23) and animal models $(18,20,30)$. Caffeine has been used in combination with NSAIDs for many decades to enhance their analgesic and anti-inflammatory effects (31-33), suggesting that caffeine may be an effective adjuvant to NSAIDs. Thus, Caff may potentiate the effects of NSAIDs by preventing the cascade of events leading to severe OIR. Further evidence of the beneficial effects of Keto/Caff coadministration is presented in Table 3. Eye opening in rodents is a key indicator of retinal maturation and development of the retinal neural circuitry $(34,35)$. Keto/Caff cotreatment in IH accelerated visual maturation and significantly shortened the caecal period compared to the placebo-treated rats exposed to IH.

Vascular growth factors are critical in angiogenesis and VEGF is in the promoting angiogenesis via activation of its receptors expressed on vascular endothelial cells. VEGF relies on IGF-I as a permissive factor which may be impaired in babies with severe ROP (36). The present study shows that the level of growth factors in the systemic circulation does not necessarily represent that in the ocular compartment. Ocular Keto had no effect on systemic VEGF or IGF, but had a moderate suppressive effect on systemic sVEGFR-1 at P14. Conversely, ocular Keto had significant effects on growth factors in the retina and choroid. Acuvail (ketorolac tromethamine ophthalmic solution $0.45 \%$ ) is a nonpreserved, isotonic ophthalmic solution with analgesic, ant-inflammatory, and antipyretic activity and contains carboxymethylcellulose (CMC), sodium chloride, and sodium citrate with a $\mathrm{pH}$ of 6.8 . The benefit of CMC is its ability to enhance ocular bioavailability and tolerability. This may account for the increased absorption and ocular penetration of the drug. The responses of growth factors to the drugs are not uniform in RA and $\mathrm{IH}$, suggesting that IH itself may interfere with drug activity. The effect of IH on retinal and choroidal HIF $_{1 \alpha}$ levels has not been previously studied. Data showed that continuous IH episodes increased $\mathrm{HIF}_{1 \alpha}$ in the retina, but not in the choroid, during IH and IHR. This response was reversed with Keto/Caff treatment during $\mathrm{IH}$, but not during IHR. However, Keto and Caff administered alone had significant suppressive effects on retinal $\mathrm{HIF}_{1 \alpha}$ which correlated with the changes in retinal VEGF. The effect of Caff on $\mathrm{HIF}_{1 \alpha}$ corroborates previous findings (37) and provides further evidence for a protective role of caffeine for prevention of VEGF overproduction and subsequent neovascularization.

Soluble VEGFR-1 (sVEGFR-1) is an endogenous negative regulator of VEGF. It acts as a VEGF "trap" by binding VEGF and preventing its availability to the membrane VEGF receptors. It was of great interest that in the placebo-treated groups at P14 and more so at P21, sVEGFR-1 levels were low to nondetectable in the retina. This finding was consistent with severe OIR production as demonstrated in $4 \mathrm{E}$ and $4 \mathrm{M}$ and suggests 
Articles | Aranda etal.

Table 5. Fold change in retinal VEGF signaling genes at P21 (recovery/reoxygenation)

\begin{tabular}{|c|c|c|c|c|c|c|c|}
\hline Genes of interest & Sal/Sal IH & Keto/Sal RA & Keto/Sal IH & Sal/Caff RA & Sal/CaffIH & Keto/Caff RA & Keto/Caff IH \\
\hline $\operatorname{cox}-2$ & $11.2 \pm 0.17$ & $-7.5 \pm 0.0001$ & $-5.3 \pm 0.0002$ & $-3.1 \pm 0.006$ & $-5.5 \pm 0.0002$ & $1.4 \pm 0.36$ & $-4.0 \pm 0.001$ \\
\hline eNOS & $1.2 \pm 0.23$ & $-9.6 \pm 0.0002$ & $-7.0 \pm 0.001$ & $-4.0 \pm 0.002$ & $-5.5 \pm 0.002$ & $1.6 \pm 0.36$ & $-3.1 \pm 0.003$ \\
\hline Mapk11 & $-1.2 \pm 0.35$ & $50.1 \pm 0.001$ & $43.6 \pm 0.0003$ & $73.9 \pm 0.0001$ & $63.1 \pm 0.0006$ & $125.8 \pm 0.008$ & $93.1 \pm 0.0007$ \\
\hline Nfat5 & $1.1 \pm 0.02$ & $-35.7 \pm 0.0001$ & $-29.9 \pm 0.0002$ & $-36.1 \pm 0.0001$ & $-27.1 \pm 0.0001$ & $-3.4 \pm 0.37$ & $-27.8 \pm 0.0002$ \\
\hline Pdgfc & $-1.2 \pm 0.21$ & $-1.6 \pm 0.58$ & $1.0 \pm 0.67$ & $1.7 \pm 0.07$ & $1.1 \pm 0.40$ & $4.2 \pm 0.36$ & $-1.3 \pm 0.96$ \\
\hline Pgf & $1.1 \pm 0.74$ & $1.4 \pm 0.29$ & $2.2 \pm 0.007$ & $3.2 \pm 0.0008$ & $2.3 \pm 0.02$ & $22.6 \pm 0.35$ & $3.7 \pm 0.05$ \\
\hline Pik3r5 & $-1.8 \pm 0.39$ & $147.7 \pm 0.0001$ & $141.1 \pm 0.0004$ & $190.0 \pm 0.003$ & $180.8 \pm 0.004$ & $410.6 \pm 0.28$ & $216.3 \pm 0.01$ \\
\hline Pla2g12a & $2.3 \pm 0.01$ & $314.3 \pm 0.0003$ & $282.8 \pm 0.0002$ & $460.3 \pm 0.0007$ & $301.9 \pm 0.002$ & $483.4 \pm 0.04$ & $258.2 \pm 0.005$ \\
\hline Plcg2 & $-1.2 \pm 0.3$ & $15.5 \pm 0.007$ & $21.6 \pm 0.0003$ & $38.9 \pm 0.0004$ & $30.1 \pm 0.0009$ & $38.9 \pm 0.21$ & $28.3 \pm 0.004$ \\
\hline VEGF-B & $1.0 \pm 0.85$ & $-12.4 \pm 0.0006$ & $-9.1 \pm 0.0007$ & $-5.0 \pm 0.001$ & $-7.3 \pm 0.0008$ & $1.7 \pm 0.36$ & $-10.4 \pm 0.0008$ \\
\hline VEGF-C & $-1.0 \pm 0.88$ & $2.3 \pm 0.09$ & $2.3 \pm 0.03$ & $2.4 \pm 0.01$ & $3.0 \pm 0.07$ & $10.2 \pm 0.36$ & $3.6 \pm 0.02$ \\
\hline VEGFR-1 & $-1.3 \pm 0.32$ & $-3.1 \pm 0.03$ & $-3.0 \pm 0.03$ & $-1.3 \pm 0.33$ & $-2.3 \pm 0.03$ & $9.8 \pm 0.36$ & $-1.1 \pm 0.91$ \\
\hline VEGFR-2 & $11.0 \pm 0.89$ & $1.1 \pm 0.31$ & $-1.0 \pm 0.89$ & $1.7 \pm 0.004$ & $1.4 \pm 0.009$ & $5.2 \pm 0.13$ & $1.4 \pm 0.02$ \\
\hline VEGFR-3 & $-2.2 \pm 0.20$ & $3.1 \pm 0.15$ & $2.2 \pm 0.55$ & $4.6 \pm 0.05$ & $3.6 \pm 0.06$ & $72.9 \pm 0.36$ & $5.8 \pm 0.06$ \\
\hline
\end{tabular}

Genes of interest are as described in Table 4. Data are compared to Sal/Sal in Room Air (RA); IH (intermittent hypoxia); IP (intraperitoneal). Minus value = downregulated. Ocular saline+IP saline (Sal/Sal); Ocular ketorolac+IP saline (Keto/Sal); Ocular saline+IP caffeine (Sal/Caff); Ocular ketorolac+IP caffeine (Keto/Caff).

that OIR resulting from IH may include in part, reductions in sVEGFR-1. Treatment with Keto, Caff, and their combination, increased retinal and choroidal sVEGFR-1, suggesting a better modulation of retinal angiogenesis and prevention or minimization of aberrant neovascularization. The increase in sVEGFR-1 was even more marked during IHR, which has consistently been shown in our model, to be associated with severe OIR. It is possible that the beneficial effects of combined Keto/Caff may be due to induction of sVEGFR-1 acting as a VEGF "trap". As an alternate therapeutic approach, it would be interesting to see if strategic pharmacologic interventions at the time of onset of reoxygenation would result in a better outcome as opposed to early postnatal treatment. Ongoing studies in our laboratory will address this issue. IGF-1 serves as a permissive factor for VEGF action (36). IGF-1 may therefore be another target for the protective effect of these drugs in the OIR. Retinal IGF-1 was significantly decreased by IH which is consistent with the notion that babies with severe OIR and oxidative injury have lower IGF-1 (36). This finding was not reflected in the serum. Instead, IH induced serum IGF-I levels and Keto cotreatment with Caff reversed the suppressive effect of IH on retinal and choroidal IGF-I.

Consistent with human ROP, our OIR model presents with more severe OIR during the IHR phase. In this report, we show a relative lack of gene response to IHR in the retinas of placebo-treated animals exposed to IH. In contrast, Keto and/ or Caff had profound effects, resulting in several fold change in many of the protective genes compared to room air placebo controls. These include upregulation of heat-shock protein (Hsp) which protects tissues from various insults and participate in the repair of damaged tissue; mitogen-activated protein kinase (MAPK)-11, also known as $p 38-\beta$ is one of the four $p 38$ MAPKs which play an important role in the cascades of cellular responses to stress; and phosphoinositide 3 kinase which is involved in preservation of photoreceptor structure and function (38); VEGFR-3; and NP-1. Studies suggest that VEGFR-3 may be upregulated in the absence of VEGF signaling (39). Therefore, upregulation of VEGFR-3 with Keto and/or Caff may be one mechanism of protection against angiogenesis. NRP-1 is a coreceptor for both VEGF and semaphorins which are involved in normal angiogenesis, axon guidance, axon repulsion and migration. It increases the potency of, and is specific for, the $V E G F_{165}$ isoform. However, there is no response to $V E G F_{165}$ in the absence of VEGFR-2 expression. Downregulated genes include nuclear factor of activated $\mathrm{T}$ cells (Nfat)-5, which is highly upregulated by hypoxia, $P K C \alpha$ which is involved in proliferation of retinal pigmented epithelial cells, and VEGF-B which is critical for VEGFR-1 binding and plasminogen activation during tissue remodeling (40).

Caff significantly decreased the risk of severe OIR, but did not prevent it completely, as was shown in the Caffeine for Apnea of Prematurity trial $(12,13)$, unless given with ketorolac. We conclude that local ophthalmic ketorolac is probably safe and has no adverse local effect on the cornea, lens nor on the conjunctiva. Keto co-administration with Caff exerts a strong synergistic or additive effect to prevent OIR in this 
rat model which closely simulates apnea of prematurity, or IH in ELGANs. Our study supports a novel therapeutic approach of combining topical ocular ketorolac (Acuvail) with systemic caffeine citrate (Cafcit) to optimize their efficacy for prevention of ROP and justifies further clinical trials. A multicenter phase IIB clinical trial (ClinicalTrials. gov:NCT02344225) is currently ongoing and will help in the design of future phase III randomized efficacy and safety clinical trials.

\section{METHODS}

All experiments were conducted according to the Association for Research in Vision and Ophthalmology Statement for the Use of Animals in Ophthalmic and Visual Research. This study was approved by the State University of New York, Downstate Medical Center Institutional Animal Care and Use Committee (protocol \#:11-10255).

\section{Experimental Design}

Timed-pregnant Sprague Dawley rats were purchased from Charles River Laboratories (Wilmington, MA). Newborn rat pups delivered on the same day were pooled and randomly assigned to expanded litters of 18 pups/litter ( 9 males and 9 females). The expanded litter size was used to simulate relative postnatal malnutrition ELGANs experience, which increases their risk for ROP. Within $2-3 \mathrm{~h}$ of birth, each pup was weighed and measured for linear growth (crown to rump length in centimeters), then exposed to IH which consisting of eight brief, clustered IH episodes per day $\left(12 \% \mathrm{O}_{2}\right)$ every $3 \mathrm{~h}$ from P0 to P14 (8 groups, $n=18 /$ group), or room air (RA, 8 groups, $n=18$ /group). Within each oxygen group (RA or IH), animals were treated with either: (i) topical ocular Keto (Acuvail, $0.45 \%$ ophthalmic solution, $0.01 \mathrm{ml}$, Allergan, CA, USA) administered subcutaneously over the eyes once per day from P5-P7 with caffeine citrate (Cafcit) loading dose $(20 \mathrm{mg} / \mathrm{kg})$ administered intraperitoneally (IP) in $0.02 \mathrm{ml}$ volume diluted in sterile normal saline) on P0, followed by maintenance doses of $5 \mathrm{mg} / \mathrm{kg} /$ day from P1 to P13. The RA groups are Keto/Caff-RA and the IH groups are Keto/Caff-IH; (ii) topical ocular Keto as described above and equivalent volume sterile normal saline administered IP from P0 to P13 (Keto/Sal-RA or Keto-Sal-IH); (iii) ocular saline once per day from P5-P7 and Caff IP from P0 to P13 (Sal/Caff-RA or Sal/Caff-IH); or (iv) ocular saline eye once per day from P5-P7 and IP saline from P0 to P13 (Sal/Sal-RA or Sal/Sal-IH), and euthanized at P14 (to determine immediate effects of $\mathrm{IH}, 4$ groups, $n=18$ pups/group) or P21 (to determine effects of recovery from IH or IHR, 4 groups, $n=18$ pups/group). The RA controls remained in normoxia from birth to P14 or birth to P21. Drug or placebo were administered using a 19 gauge needle attached to a $0.5 \mathrm{ml}$ tuberculin syringe. Animals in the IHR group remained in RA until P21 with no further drug or saline treatment. Refer to Supplementary Figure S4 online for a flow chart of the experimental design. To administer topical ocular Acuvail, a tent was made by gently pinching the skin above the eyes and inserting the needle into the tent so that there is no contact with the cornea. Acuvail is a new and improved ocular formulation that has minimal adverse effects such as pain or eye irritation compared to Acular.

\section{Intermittent Hypoxia (IH) Cycling.}

Animals randomized to IH were placed with the dams in specialized oxygen chambers attached to an oxycycler (BioSpherix, New York) as previously described (25-29). The IH profile consisted of keeping the rat pups in hyperoxia (50\%) with intermittent burst of three clustered episodes of hypoxia (12\%) each $10 \mathrm{~min}$ apart every $3 \mathrm{~h}$ as described previously from our laboratory $(27,29)$. Thus, the neonatal pups were subjected daily to eight of these clustered episodes of intermittent hypoxia during hyperoxia to simulate a preterm newborn having repeated desaturations while on $50 \%$ inspired oxygen. This clustering design has been shown to produce a severe form of OIR in neonatal rats (25-29). Refer to Supplementary Figure S5 online for the intermittent hypoxia profile.

\section{Sample Collection}

For ELISA assays, blood samples were collected in sterile Eppendorf tubes, placed on ice and allowed to clot for $30 \mathrm{~min}$ prior to centrifugation and collection of serum. There were 10 serum samples ( 5 males and 5 females) per group. Eyes were enucleated and rinsed in ice-cold phosphate-buffered saline ( $\mathrm{pH}$ 7.4) on ice. For enzyme immunoassays (EIA), retinas and choroids were excised and processed as previously described (25-29). To obtain enough tissue, retinas and choroids were pooled for a total of six samples each (three males and three females). For ADP staining of the retinal flatmounts, whole eyes were placed in $4 \%$ paraformaldehyde for $90 \mathrm{~min}$ prior to flatmounting. For histopathology of the corneas and retinal layers, whole eyes were placed in Hartmann's fixative and sent to New York University Experimental Pathology Histology Core Laboratory for $\mathrm{H} \& \mathrm{E}$ staining.

\section{VEGF, sVEGFR-1, IGF-I}

EIA assays of VEGF, sVEGFR-1 and IGF-I in the retinas and choroids were determined as previously described (26-28). Levels were standardized using total cellular protein levels according to the Bradford method (BioRad Laboratories, Hercules, CA).

\section{Hypoxia-Inducible Factor (HIF) $1 \alpha$}

HIF $_{1 \alpha}$ levels were determined in the retina and choroid using a commercially-available EIA duo set kits from R \& D Systems (Minnealpolis, MN, USA). Levels were standardized using total cellular protein levels according to the Bradford method.

\section{8-isoPGF $2 \alpha$}

8 -isoPGF ${ }_{2 \alpha}$ HIF $_{1 \alpha}$ levels in the retinas and choroids were determined using a commercially-available EIA kits from Cayman Chemicals, Ann Arbor, MI. Levels were standardized using total cellular protein levels according to the Bradford method.

\section{Retinal ADPase Staining}

ADPase staining of the retinas and computer imaging were carried out as previously described (25-29). Digital images of the whole flattened, stained retinas were captured at $4 \times$ magnification for assessment of neovascularization using an Olympus BX53 microscope, DP72 digital camera, and CellSens imaging software (Olympus, Center Valley, PA), attached to a Dell Precision T3500 computer (Dell, Round Rock, TX).

\section{Retinal H \& E Staining}

Eyes were enucleated, rinsed in phosphate-buffered saline (PBS), fixed in Hartmann's fixative and sent to New York University Experimental Pathology Histology Core Laboratory, NY, NY for H \& E staining. Images were captured at $40 \times$ (Figure 5, b1) and $60 \times$ (Figure 5, b2) magnification using an Olympus BX53 microscope, DP72 digital camera, and CellSens imaging software attached to a Dell Precision T3500 computer.

\section{Immunofluorescence}

Immunofluorescence staining for HIF, VEGF, VEGFR-2 and COX-2 were carried out using primary antibodies purchased from Santa Cruz Biotechnology (Dallas, TX) and Alexa Fluor fluorescent secondary antibodies purchased from Life Technologies (Grand Island, NY), and conducted according to the manufacturer's recommendations. Images were captured at 40× magnification using an Olympus BX53 microscope, DP72 digital camera, and CellSens imaging software attached to a Dell Precision T3500 computer.

\section{Real-Time Polymerase Chain Reaction}

Total retinal RNA was extracted as previously described $(25,28,29)$. To identify genes that are affected by IH, real-time PCR arrays were carried out in quadruple using the rat VEGF Signaling PCR array systems (SABiosciences, Frederick, MD) using a BioRad IQ5 real-time instrument.

\section{Statistical Analysis}

One-way ANOVA was used to determine differences among the treated groups for normally-distributed data, and Kruskal-Wallis test 
was used for non-normally-distributed data following Bartlett's test for equality of variances. Post hoc analysis was performed using the Tukey, Bonferoni and Student-Newman-Keuls tests for significance. To compare data between RA and IH groups, unpaired $t$-test was employed for normally distributed data and Mann Whitney $U$-tests were used for non-normal data following Levene's test for equality of variances. Significance was set at $P<0.05$ and data are reported as mean \pm SEM. All analyses were two-tailed and performed using SPSS version 16.0 (SPSS, Chicago, IL). Graphs were prepared using GraphPad Prism ver. 5 software (GraphPad, San Diego, CA).

\section{STATEMENT OF FINANCIAL SUPPORT}

This work was supported by the National Institutes of Health -Eunice Kennedy Shriver National Institute of Child Health \& Human Development, Bethesda, MD, Grant \#1U54HD071594.

\section{SUPPLEMENTARY MATERIAL}

Supplementary material is linked to the online version of the paper at http:// www.nature.com/pr

Disclosures: The authors have nothing to disclose.

\section{REFERENCES}

1. Hellström A, Smith LE, Dammann O. Retinopathy of prematurity. Lancet 2013;382:1445-57.

2. Smith LE. Pathogenesis of retinopathy of prematurity. Growth Horm IGF Res 2004;14 Suppl A:S140-4.

3. Chow LC, Wright KW, Sola A; CSMC Oxygen Administration Study Group. Can changes in clinical practice decrease the incidence of severe retinopathy of prematurity in very low birth weight infants? Pediatrics 2003;111:339-45.

4. Martin RJ, Wang K, Köroğlu O, Di Fiore J, Kc P. Intermittent hypoxic episodes in preterm infants: do they matter? Neonatology 2011;100: 303-10.

5. Di Fiore JM, Kaffashi F, Loparo K, et al. The relationship between patterns of intermittent hypoxia and retinopathy of prematurity in preterm infants. Pediatr Res 2012;72:606-12.

6. York JR, Landers S, Kirby RS, Arbogast PG, Penn JS. Arterial oxygen fluctuation and retinopathy of prematurity in very-low-birth-weight infants. J Perinatol 2004;24:82-7.

7. Di Fiore JM, Bloom JN, Orge F, et al. A higher incidence of intermittent hypoxemic episodes is associated with severe retinopathy of prematurity. J Pediatr 2010;157:69-73.

8. Mintz-Hittner HA, Kennedy KA, Chuang AZ; BEAT-ROP Cooperative Group. Efficacy of intravitreal bevacizumab for stage 3+ retinopathy of prematurity. N Engl J Med 2011;364:603-15.

9. Mintz-Hittner HA. Treatment of retinopathy of prematurity with vascular endothelial growth factor inhibitors. Early Hum Dev 2012;88:937-41.

10. Moshfeghi DM, Berrocal AM. Retinopathy of prematurity in the time of bevacizumab: incorporating the BEAT-ROP results into clinical practice. Ophthalmology 2011;118:1227-8.

11. Hård AL, Hellström A. On safety, pharmacokinetics and dosage of bevacizumab in ROP treatment - a review. Acta Paediatr 2011;100:1523-7.

12. Aranda JV, Beharry K, Valencia GB, Natarajan G, Davis J. Caffeine impact on neonatal morbidities. J Matern Fetal Neonatal Med 2010;23 Suppl 3: $20-3$.

13. Schmidt B, Roberts RS, Davis P, et al.; Caffeine for Apnea of Prematurity Trial Group. Caffeine therapy for apnea of prematurity. N Engl J Med 2006;354:2112-21.

14. Schmidt B, Roberts RS, Davis P, et al.; Caffeine for Apnea of Prematurity Trial Group. Long-term effects of caffeine therapy for apnea of prematurity. N Engl J Med 2007;357:1893-902.

15. Sahir N, Evrard P, Gressens P. Caffeine induces sonic hedgehog gene expression in cultured astrocytes and neurons. J Mol Neurosci 2004;24:201-5.

16. Merighi S, Benini A, Mirandola P, et al. Caffeine inhibits adenosineinduced accumulation of hypoxia-inducible factor-1alpha, vascular endothelial growth factor, and interleukin-8 expression in hypoxic human colon cancer cells. Mol Pharmacol 2007;72:395-406.

17. Liu WH, Chang LS. Caffeine induces matrix metalloproteinase-2 (MMP2) and MMP-9 down-regulation in human leukemia U937 cells via Ca2+l ROS-mediated suppression of ERK/c-fos pathway and activation of p38 MAPK/c-jun pathway. J Cell Physiol 2010;224:775-85.

18. Beharry KD, Modanlou HD, Hasan J, et al. Comparative effects of early postnatal ibuprofen and indomethacin on VEGF, IGF-I, and GH during rat ocular development. Invest Ophthalmol Vis Sci 2006;47:3036-43.

19. Hardy P, Dumont I, Bhattacharya M, et al. Oxidants, nitric oxide and prostanoids in the developing ocular vasculature: a basis for ischemic retinopathy. Cardiovasc Res 2000;47:489-509.

20. Sharma J, Barr SM, Geng Y, Yun Y, Higgins RD. Ibuprofen improves oxygen-induced retinopathy in a mouse model. Curr Eye Res 2003;27:309-14.

21. Yeh TF, Raval D, Pyati S, Pildes RS. Retinopathy of prematurity (ROP) and indomethacin therapy in premature infants with patent ductus arteriosus (PDA). Prostaglandins 1983;25:385-91.

22. Goldman RD, Spierer A, Zhurkovsky A, Kwint J, Schwarcz M, Ben Simon GJ. Retinopathy of prematurity in very low birth weight infants and the potential protective role of indomethacin. Ophthalmic Surg Lasers Imaging 2010;41:41-7.

23. Avila-Vazquez M, Maffrand R, Sosa M, et al. Treatment of retinopathy of prematurity with topical ketorolac tromethamine: a preliminary study, BMC Pediatr 2004; 7:4-15.

24. Reynolds JD. The management of retinopathy of prematurity. Paediatr Drugs 2001;3:263-72.

25. Jivabhai Patel S, Bany-Mohammed F, McNally L, et al. Exogenous Superoxide Dismutase Mimetic Without Scavenging H2O2 Causes Photoreceptor Damage in a Rat Model for Oxygen-Induced Retinopathy. Invest Ophthalmol Vis Sci 2015;56:1665-77.

26. Tu C, Beharry KD, Shen X, et al. Proteomic profiling of the retinas in a neonatal rat model of oxygen-induced retinopathy with a reproducible ion-current-based MS1 approach. J Proteome Res 2015;14:2109-20.

27. Beharry KD, Cai CL, Sharma P, et al. Hydrogen peroxide accumulation in the choroid during intermittent hypoxia increases risk of severe oxygen-induced retinopathy in neonatal rats. Invest Ophthalmol Vis Sci 2013;54:7644-57.

28. Brock RS, Gebrekristos BH, Kuniyoshi KM, Modanlou HD, Falcao MC, Beharry KD. Biomolecular effects of JB1 (an IGF-I peptide analog) in a rat model of oxygen-induced retinopathy. Pediatr Res 2011;69:135-41.

29. Coleman RJ, Beharry KD, Brock RS, Abad-Santos P, Abad-Santos M, Modanlou HD. Effects of brief, clustered versus dispersed hypoxic episodes on systemic and ocular growth factors in a rat model of oxygeninduced retinopathy. Pediatr Res 2008;64:50-5.

30. Nandgaonkar BN, Rotschild T, Yu K, Higgins RD. Indomethacin improves oxygen-induced retinopathy in the mouse. Pediatr Res 1999;46:184-8.

31. Fiebich BL, Lieb K, Hüll M, et al. Effects of caffeine and paracetamol alone or in combination with acetylsalicylic acid on prostaglandin $\mathrm{E}(2)$ synthesis in rat microglial cells. Neuropharmacology 2000;39:2205-13.

32. Dooley JM, Gordon KE, Wood EP, Brna PM, MacSween J, Fraser A. Caffeine as an adjuvant to ibuprofen in treating childhood headaches. Pediatr Neurol 2007;37:42-6.

33. López JR, Domínguez-Ramírez AM, Cook HJ, et al. Enhancement of antinociception by co-administration of ibuprofen and caffeine in arthritic rats. Eur J Pharmacol 2006;544:31-8.

34. Binns KE, Salt TE. Post eye-opening maturation of visual receptive field diameters in the superior colliculus of normal- and dark-reared rats. Brain Res Dev Brain Res 1997;99:263-6.

35. Robinson SR, Dreher B. The visual pathways of eutherian mammals and marsupials develop according to a common timetable. Brain Behav Evol 1990;36:177-95.

36. Hellström A, Engström E, Hård AL, et al. Postnatal serum insulin-like growth factor I deficiency is associated with retinopathy of prematurity and other complications of premature birth. Pediatrics 2003;112: 1016-20. 
37. Merighi S, Benini A, Mirandola P, et al. Caffeine inhibits adenosineinduced accumulation of hypoxia-inducible factor-1alpha, vascular endothelial growth factor, and interleukin-8 expression in hypoxic human colon cancer cells. Mol Pharmacol 2007;72:395-406.

38. Rajala RVS. Phosphoinositide 3-kinase signaling in the vertebrate retina. J Lipid Res 2010;51:4-22.

39. Benedito R, Rocha SF, Woeste M, et al. Notch-dependent VEGFR3 upregulation allows angiogenesis without VEGF-VEGFR2 signalling. Nature 2012;484:110-4.

40. Olofsson B, Korpelainen E, Pepper MS, et al. Vascular endothelial growth factor B (VEGF-B) binds to VEGF receptor-1 and regulates plasminogen activator activity in endothelial cells. Proc Natl Acad Sci USA 1998;95:11709-14.
This work is licensed under a Creative Commons Attribution-NonCommercial-NoDerivs $\quad \mathbf{4 . 0}$ International License. The images or other third party material in this article are included in the article's Creative Commons license, unless indicated otherwise in the credit line; if the material is not included under the Creative Commons license, users will need to obtain permission from the license holder to reproduce the material. To view a copy of this license, visit http://creativecommons.org/ licenses/by-nc-nd/4.0/

(c) JV Aranda et al. (2016) 